\title{
SOME ASPECTS OF HEART DISEASE IN RELATION TO RHEUMATISM
}

\author{
By BASIL PARSONS-SMITH, M.D., F.R.C.P.
}

(Physician to the National Heart Hospital)

In its modern conception acute rheumatism is regarded as a general disease which attacks certain organs, serous membranes and tissues of the body, the heart, more often than not, being implicated in the process.

Though somewhat variable in the extent of their involvement, the myocardium, the endocardial structures, and the pericardium may all be affected by the disease and the complex disability so produced is referred to under the collective title rheumatic carditis.

The condition is essentially a development of youth and adolescence (Thayer, I93I); it is responsible for 90 per cent. of all heart diseases in children, and it supervenes in approximately 30 per cent. of adults suffering from acute rheumatism (Scherf and Boyd, I939); it frequently accounts for the relapses which are relatively common in rheumatic children and, though constitutional symptoms usually occur to confirm the diagnosis, it may proceed without any obvious manifestations of muscular involvement or arthritis.

Certain definite variations have been recognized in the manifestations of acute rheumatism as they appear at different periods of life (Conner, I937); primary attacks in adults are seldom followed by valvular disease; in children, on the other hand, carditis in some form is practically an inevitable development and it has been estimated that in the elementary schools of Great Britain there are some 40,000 cases of rheumatic heart disease (Schlesinger, Signy and Amies, I935).

The aetiology of rheumatism has been the subject of an intensive research during the past fifty years and, although the results to date fail to admit of final conclusions, it is generally agreed that valuable advances have been achieved which give high promise that the problem is gradually nearing solution.

Assuming acute rheumatism to be the outcome of a general infection, and, having in mind the uniform character of its morbid anatomy, it is not surprising that numerous investigations have been carried out with a view to isolating a microbe specific for the disease.

The theory of a streptococcal infection has for many years been the basis of clinical and experimental study both in this country and abroad; Poynton and Paine (I900) isolated a diplostreptococcus from the heart valves, pericardium, tonsils, nodules, etc., in a series of cases of rheumatic fever, and rabbits, inoculated with these diplococci, developed characteristic lesions of the rheumatic type; the authors, however, were not prepared to state that the diplococci, which incidentally were later classed as non-haemolytic streptococci of the viridans type, were invariably present in cases of rheumatic fever, nor did they claim that they were the sole cause of the disease. In more recent times the streptococcal hypothesis has been strongly supported by Miller (I938), who associates acute rheumatism with a haemic infection by non-pyogenic streptococci of the viridans type which more particularly tends to attack the children of the poorer classes, those living in the industrial towns who attend school under compulsion and those suffering from tonsillar sepsis. The streptococci circulating in the coronary vessels are, according to Miller, responsible for the proliferative tissue reaction originally described by Aschoff (1906), whose researches can be briefly epitomised as follows:-

\begin{abstract}
"In true rheumatic affections peculiarly submiliary nodules tend to develop both in the interstitial connective tissue, especially in that surrounding the arteries, and in the subendocardial connective tissue layers; the nodules consist of peculiar large elements containing structures looking like giant nuclei; many of the cells are arranged radially about a centre as in tubercle, but the cells of the rheumatic nodule greatly exceed the epithelioid cells of tubercle in size; besides these large cells there are also lymphatic and neutrophile leucocytes as well as some containing eosinophile granules; these infiltrations of small cells are continued as streaks into the surrounding tissues; in the great majority of valve disease cases there is no fresh interstitial inflammation but remnants of the rheumatic nodules remain in the shape of more or less numerous perivascular fibrous nodules; from these nodules arise large areas of anaemic necrosis, the so-called infarct indurations which constitute one of the more serious intrinsic lesions in cases of rheumatic carditis."
\end{abstract}

A further alternative theory was advanced by Schlesinger and his colleagues (I935), whose investigations favoured a virus as the infective agent in rheumatic disease. They isolated pure suspensions of virus elementary bodies from the pericardial and pleural exudates of patients 
suffering from acute rheumatism and they were able to demonstrate that these bodies were specifically agglutinated by the sera of patients suffering from rheumatic infection. The authors were of opinion therefore that acute rheumatism should be regarded as a virus disease and they suggested that, following a preliminary infection by haemolytic streptococci, the natural resistance was sufficiently reduced for the virus to gain access to the body, or to activate the virus if it happened to be already present in the tissues in latent form.

It is problematical whether or not the virus hypothesis of rheumatism will receive substantial confirmation later, but, in the meantime, there is general agreement that acute rheumatism is probably associated with several different types of haemolytic streptococci, and that its occurrence may to some extent depend upon certain constitutional peculiarities of the subject (Poynton and Schlesinger, I937). In this connection the possibility of an allergic factor has been considered. Alterations in the heart such as are found in rheumatic myocarditis and endocarditis can be produced in animals by sensitisation with certain proteins or by the injection of certain strongly acting toxins (Scherf and Boyd, I939), and such evidence supports the conception that acute rheumatism may be an allergic disease resulting from the development of hypersensitivity to the products of various strains of streptococci.

The clinical picture and diagnostic features of acute rheumatic carditis are problems in the investigation of which children and young adolescents offer the most profitable field of approach.

Vague symptoms of general debility occur more often than not for weeks or months prior to the development of the disease in diagnosable form. The child looks ill and pale, and the level of nutrition may be obviously impaired, in some cases to the degree of actual emaciation. Lassitude, lack of energy and enthusiasm, irritability, restlessness and a mental inertia, are all in varying degree characteristic premonitory symptoms, and otherwise the child's subjective complaints usually include fatigue, loss of appetite, intermittent pains in the limbs, and frequently recurring colds and sore throats.

The close association between rheumatism and tonsillitis has been strongly emphasised and in the large majority of cases an acute infection, of which the dominant organism is a haemolytic streptococcus, precedes the invasion stage of acute carditis (Coburn, I936). The latter is characterised by a variety of clinical signs the more important of which include diffusion and enfeeblement of the apex impulse, tachycardia, which persists during sleep, blurring of the first heart sound and pericardial friction. In addition it is customary to find a persistently raised temperature during the active stages of rheumatic carditis, a considerably raised blood sedimentation rate, varying degrees of hypochromic anaemia, and a leucocyte count in an average case of 10,000 to 12,000 per c.mm.

As a general rule the degree of the symptoms depends upon the severity of the infection and the extent to which the myocardium is involved in the process; further indications may however appear in the more serious grades of the disease. These include marked pallor with deep cyanosis, urgent breathlessness, a persistently fast and irregular pulse, sustained pyrexia, restlessness, delirium, cardiac vomiting and pericarditis.

The above-mentioned complex of symptoms varies in its grade of severity according to the gravity of the myocarditis factor which occurs to a greater or lesser extent in all cases of cardiac rheumatism. Since moreover the functions of the myocardium have been analysêd in detail by electrocardiographic methods it is now possible to assess the myocardial factor with a considerable degree of accuracy. Cohn and Swift (I929) investigated 37 patients suffering from acute rheumatism and of these 35 showed cardiographic evidence of rheumatic carditis. The changes included alterations in auriculo-ventricular conduction time, discrepancies in the ventricular portion of the curves and all the known forms of arrhythmia. A prolonged P.R. interval was noted in $3 \mathrm{I}$ of the patients and, though the majority of these had varying degrees of fever, the conduction delay had no specific relation to the height of the temperature. The authors were of opinion that electrocardiography was the outstanding method of determining whether and when the heart was involved in acute rheumatism.

Levy and Turner (I929) recorded a prolonged P.R. interval in $27 \cdot 8$ per cent. of a series of rheumatic fever cases and they noted characteristic post-mortem lesions in the auriculo-ventricular bundle which included cellular infiltration and oedema in the acute stages and scar tissue later. Their final dictum was as follows: "A prolonged P.R. interval or heart block in a person under 35 , who is not syphilitic and who has not taken digitalis, affords presumptive evidence of the presence of rheumatic carditis." 
Rothschild and his colleagues (I926) examined the cardiographic records of 65 cases of acute rheumatism. Sixty one of these cases showed evidence of carditis in the shape of conduction disturbances, or rhythm changes or deviations in the contour of the ventricular complexes. Delayed conduction from the auricles to the ventricles occurred in 23 cases of the group, and the authors stressed transient heart-block in all its forms, e.g. prolongation of the P.R. interval, ventricular intermissions and partial or complete dissociation, as the most frequent discrepancy in acute rheumatic carditis. The ventricular portion of the cardiogram showed characteristic abnormalities in 52 of the 65 cases, the more outstanding of these abnormalities being as follows:-

(I) Notching, widening and alterations in amplitude and direction of the $\mathrm{R}$ or $\mathrm{S}$ waves.

(2) Failure of the $\mathrm{R}$ waves to reach the iso-electric line and the development of an upward curve occupying the whole of the R.T. period.

(3) Depression or inversion of the $T$ waves.

The authors correlated these electrical changes with collateral oedema and local ischatmia in the vicinity of Aschoff bodies, these lesions being widespread both in the myocardium and in the sub-endocardial fibres of the Purkinje system. They regarded myocarditis as a sine qua non in practically every case of acute rheumatism, and they emphasised the value of electrocardiograms as a means of detecting cardiac invasion, the first clue to the presence of the disorder being possibly some form of heart block or abnormality in the pattern of the ventricular complexes.

In the light of the above investigations it may be assumed that, quite apart from characteristic symptoms and clinical signs, rheumatic myocarditis can be evaluated with a considerable degree of accuracy by graphic methods. Such was also the expressed opinion of Parkinson and his colleagues (I9I9-20) as the result of their analysis of 50 cases of acute rheumatism. They concluded that the presence of sinus arrhythmia was no proof that the heart had escaped infection, but that other forms of irregularity, e.g. premature auricular contractions, auricular fibrillation, auricular flutter, also acute heart block, which incidentally was noted in 30 per cent. of their cases, were indicative of acute myacarditis. These authors also suggested that the degree of enlargement of the heart during an attack of acute rheumatism had been exaggerated and it is interesting to note that the modern teaching is confirmatory in this respect, that enlargement, if it occurs at all in rheumatic carditis, is likely to be relatively slight.

It remains to consider two further manifestations of acute rheumatism, viz. pericarditis and endocarditis. The former of these is frequently an elusive development, although in point of fact it is, in some form or another, an exceedingly common complication of the disease. It may develop so insidiously that it escapes diagnosis entirely or that it is only recognised by accident. Otherwise a variety of characteristic signs may proclaim its presence; the temperature, which may have shown a tendency to decline to some extent, rises again, there is persistent tachycardia, the patient complains of palpitation and pain, both intensified by pressure and by. the act of coughing, and there may be a characteristic friction rub in the praecordial region. Subsequently there may be evidence of a pericardial exudate which as a rule manifests its presence proportionately to the rate at which the fluid formation collects. An effective increase in the pericardial pressure materially prejudices the circulatory mechanics for the venous return to the heart is thereby obstructed, the output per beat is necessarily diminished, and the blood pressure level declines. Under such circumstances venous stasis, cyanosis and pallor are constantly present in varying degree and other obvious features include orthopnoea, substernal discomfort and an unproductive cough, together with insomnia and persistent restlessness. The area of cardiac dullness is increased in all directions, its left lateral limit extending as a rule an appreciable distance beyond the region of the apex impulse. In cases moreover of massive effusion there may be an extensive area of percussion dullness posteriorly as the result of compression atelectasis at the base of the left lung; fluoroscopic examination reveals a large globular heart shadow with clear-cut borders which are otherwise peculiar in that their range of mobility is relatively much diminished or quite frequently is imperceptible to naked eye inspection.

Endocarditis is with good reason regarded as one of the major disabilities of rheumatic carditis. It occurs with grim regularity in the large majority of affected children, and there is more than an even chance of its development in the acute rheumatic infections of adults. More often than not its diagnosis cannot be made with any degree of confidence in the early stages of the disease, and months, possibly years, may need to elapse before definite evidence is forth- 
coming in the shape of valvular discrepancies. The invasion period may however include certain definite clinical hints which warrant reasonably strong suspicions of endocardial involvement; the following are especially noteworthy in this respect:-

(I) Persistent pyrexia, tachycardia and the appearances of grave illness in a patient who otherwise may have been making an uncomplicated recovery from an attack of acute rheumatism.

(2) An increased sedimentation rate of the red blood cells.

(3) The presence of a leucocytosis.

(4) The appearance of an endocardial murmur diastolic in time.

Whether or not these early clinical suspicions may ultimately be confirmed in fact, it will obviously be necessary to keep patients with such suggestive signs under prolonged supervision always bearing in mind the very insidious course that rheumatic endocarditis pursues. As a general rule the condition is confined to the valves and the chordae tendineae on the left side of the heart and, although serious harm is seldom done in the acute stage, this may be followed by extensive new formations of connective tissue which deform the valve structures and impair their normal range of activity.

Of the rheumatic valvular lesions an outstanding importance attaches to aortic regurgitation and to mitral stenosis. Although recognisable at times in the very early stages of its development, aortic regurgitation more often than not originates in a singularly insidious fashion, and the disease, with all its typical auscultatory and peripheral signs, is frequently discovered by accident in the course of a routine medical examination. That such is true is moreover not surprising, for in the large majority of cases the valvular defect is so successfully compensated that patients may continue for years a routine of unrestricted activity, enjoying in the meantime an absolute immunity from symptoms suggestive of circulatory embarrassment. Various factors combine to establish compensation at an adequate level in rheumatic aortitis, and of these an efficient myocardial function is pre-eminent. Unlike syphilis which, more often than not, implicates the orifices of the coronary vessels inducing thereby varying degrees of myocardial ischaemia, rheumatic aortitis is usually associated with localised and relatively insignificant lesions in the heart muscle. As with other valvular lesions therefore the prognosis in cases of aortic regurgitation depends chiefly upon the state of the myocardium, the reserve of which can be assessed as a rule by careful enquiry into the presenting signs, symptoms and cardiographic records. Apart from complications the reserve capacity of the heart muscle is capable of balancing the aortic leak indefinitely, but decompensation may occur at any time if intercurrent developments, e.g. acute infections, abnormal rhythms, anaemia, pregnancy, obesity and such like, occur to prejudice the natural progress of the disease. The first symptomatic evidence of failure in aortic regurgitation is breathlessness, which not only occurs spontaneously when the patient is resting but also materially lowers his range of tolerance for physical activity. The disability is indicative of left ventricular embarrassment and back-pressure stasis in the pulmonary circuit, the congested vessels of which originate vagal reflexes to the respiratory centre. As a general rule the orthopnoea of aortic disease, the paroxysmal breathlessness and the impaired vital capacity, all vary in proportion to the degree of the pulmonary congestion, and all these symptoms are to some extent relieved if right ventricular failure subsequently ensues, as evidenced by a rise in the systemic venous pressure, enlargement of the liver and general dropsy. As failure proceeds further evidence of errors in the circulatory mechanics is available; the circulation time is prolonged; pulmonary oedema persists; there is usually a sustained tachycardia, possibly a gallop rhythm, also a pulsus alternans and the electrocardiogram is usually confirmatory of myocardial incompetence. Two complications are especially worthy of mention:-

(I) Infective endocarditis which frequently supervenes as a terminal event in rheumatic aortitis, and

(2) Angina pectoris, a symptomatic manifestation of myocardial ischaemia due to illsustained diastolic pressure in the coronary vessels and in certain cases an indication of myocardial infarction without thrombotic occlusion of the coronary vessels (Friedberg and Horn, I939). 
In contradistinction to aortic disease the symptoms of decompensation in mitral stenosis develop relatively early, for the primary strain of the lesion falls on the left auricle the thin walls of which are only capable of very moderate degrees of hypertrophy. As the valvular obstruction proceeds the back pressure stasis in the left auricle gradually involves the pulmonary circuit, as evidenced by a progressive decline in the vital capacity, breathlessness on exertion, cough and a tendency to haemoptysis. The physical signs of fully developed mitral stenosis are as a rule unmistakable: a presystolic thrill and presystolic murmur, both of which can best be recognised in the recumbent position after exercise, and possibly a mid-diastolic rumble; an accentuated first sound due partly to changes in the mitral valve, partly also to overaction of the right ventricle and to underfilling of the left ventricle; accentuation of the second sound and a systolic murmur at the pulmonary base, both indicative of dilatation of the pulmonary artery and an increased level of pressure in the pulmonary circuit; signs significant of right ventricular hypertrophy in the shape of diffuse praecordial pulsations usually felt best to the left of the sternum and extending outwards and upwards to the region of the pulmonary conus; a small pulse which tends to diminish in volume as the grade of the stenotic process gradually increases; varying degrees of cyanosis, possibly with clubbing of the fingers and polycythaemia, each an obvious manifestation of defective oxygenation resulting from stasis and sclerosis in the pulmonary vessels

Of the above signs, two, viz. the presystolic murmur and the accentuated second sound at the pulmonary base, are subject to modification in later stages of the disease. The presystolic murmur disappears entirely when auricular fibrillation develops, and also in certain patients with aneurysmal dilatation of the left auricle. The second sound at the pulmonary base diminishes in intensity as the right heart progressively dilates, and at the same time the significant signs of tricuspid incompetence appear, viz. a blowing systolic murmur at the tricuspid region, diffuse pulsation at the lower end of the sternum and in the epigastric region, dilatation of the right auricle, systolic pulsation in the distended jugular vessels and systolic pulsation of the liver.

Except in its early stage of pure hypertrophy of the left auricle, the radiological appearances of mitral stenosis are clearly defined; the first appreciable signs of the disability are dilatations of the left auricle and of the pulmonary artery, the former seen best in the first oblique position as an oval swelling projecting backwards into the middle third of the posterior cardiac space, the latter in the antero-posterior view at the left base of the great vessel shadow. In its more advanced stages the heart rotates on its vertical axis owing to dilatation of the right chambers, and X-ray examination reveals the features of so-called mitralisation in their full form. The dilated pulmonary conus expands the spread of the cardiac waist, thus forming the upper segment of the left heart border which otherwise extends almost perpendicularly downwards to the apex region and is chiefly formed by the margin of the dilated right ventricle; the right border of the heart, frequently a composite shadow composed of the-left as well as the right auricle, extends well into the right lung field; the lungs show varying degrees of congestion both peripherally and at their roots.

\section{REFERENCES}

ASCHOFF, L. (1906) Brit. Med.J. 2. I103.

COBURN, A. F. (1936) Lancet. 11. ro25.

COHN, A. E., and SWIFT, H. F. (1929) Jour. Exp. Med. 39. 1.

CONNER, L. A. (1937) New Eng. J. Med. 217. 503.

DUCKETT JONES, T. (1938) Am. J. Publ. Health. 28. 637-643.

FRIEDBERG, C. K., and HORN, H. (1939) J.A.M.A. 112.1675.

LEVY, R. L., and TURNER, K. B. (1929) Arch. Int. Med. 43.267.

MILLER, R. (1938) The British Encyclopaedia of Medical Practice, London. Vol. X. 639.

PARKINSON, J., GOSSE, A. H., and GUNSON, E. B. (1919-1920) Quart. J. Med. XIII. 363.

POYNTON, F. J., and PAINE, A. (1900) Lancet. 11. 86r.

POYNTON, F. J., and SCHLESINGER, B. (1937) Recent Advances in the Study of Rheumatism, London.

ROTHSCHILD, M. A., SACKS, B., and LIBMAN, E. (1926) Amer. Heart J. 2. 356.

SCHERF, D., and BOYD, L. J. (1939) Cardiovascular Diseases, London.

SCHLESINGER, B., SIGNY, A. G., and AMIES, C. R. (1935) Lancet. 1. II45.

THAYER, W. S. (I93I) Ann. Int. Med. 5. 247.

WARNER, E. C. (1937) Med. Press. 194. 423. 